\title{
Technique murale picturale
}

Auxerre, Centre d'études médiévales, 15-16 mai 2009

\section{Claude Coupry}

\section{(2) OpenEdition}

Journals

Édition électronique

URL : https://journals.openedition.org/cem/11079

DOI : $10.4000 /$ cem. 11079

ISSN : 1954-3093

Éditeur

Centre d'études médiévales Saint-Germain d'Auxerre

Édition imprimée

Date de publication : 15 août 2009

Pagination : 251-255

ISSN : $1623-5770$

Référence électronique

Claude Coupry, «Technique murale picturale », Bulletin du centre d'études médiévales d'Auxerre | BUCEMA [En ligne], 13 | 2009, mis en ligne le 04 septembre 2009, consulté le 22 septembre 2022. URL : http://journals.openedition.org/cem/11079; DOI : https://doi.org/10.4000/cem.11079

Ce document a été généré automatiquement le 22 septembre 2022.

\section{(c) $)(1)(2)$}

Creative Commons - Attribution - Pas d'Utilisation Commerciale - Partage dans les Mêmes Conditions 4.0 International - CC BY-NC-SA 4.0

https://creativecommons.org/licenses/by-nc-sa/4.0/ 


\title{
Technique murale picturale
}

\author{
Auxerre, Centre d'études médiévales, 15-16 mai 2009
}

\section{Claude Coupry}

1 Après des études remarquables publiées, entre autres, dans Peindre à Auxerre au Moyen Âge, IX $X^{\mathrm{e}}-X I V^{\mathrm{e}}$ siècles, l'étude technique et analytique des peintures murales n'avait pas donné lieu à de nouvelles recherches méthodologiques en dépit des travaux de terrain (Souvigny, Stavelot...) où l'archéologie du bâti avait permis des rencontres entre archéologues, historiens de l'art et restaurateurs particulièrement impliqués pour la période qui nous intéresse, de la basse Antiquité au haut Moyen Âge.

2 Cet atelier nous a donné l'opportunité de rassembler quelques spécialistes du domaine afin de mettre en commun les différentes approches, observations et interrogations, et de dégager des directions de réflexion et de recherche.

3 Le programme montre la diversité des interventions. À partir du constat des recherches présentées lors d'un colloque en 1996 «Roman Wall Painting, Materials, Techniques, Analysis and Conservation ", Michel Fuchs interroge sur la place donnée actuellement aux analyses dans les recherches sur les peintures. La matériauthèque du Centre d'étude des peintures murales romaines (Soissons), présentée par Florence Monier, donne un support matériel de comparaison à partir de fragments répertoriés, plus d'un millier provenant de 120 sites, et pose le problème des critères de choix des fragments ainsi que le mode d'utilisation concret de cet ensemble. La technique de la fresque a été au cœur de l'approche d'archéologie expérimentale de Stéphane Treilhou, étayée par une lecture approfondie des textes techniques antiques. Ses résultats débouchent sur des questionnements fondamentaux sur la nature même de la fresque et sa composition.

4 Les interventions suivantes correspondent à des études soit de sites soit centrées sur des matériaux. Paulette Hugon a montré la méthodologie adoptée pour l'étude de Saint-Savin, avec les interventions multiples et concertées des différents spécialistes et les apports considérables auxquels elle a conduit. Rosalie Godin apporte le point de vue de la restauratrice et insiste sur la collecte d'échantillons, 
capitale pour des interprétations pertinentes, et illustrée sur le site de l'Hôtel Saint-Jean à Toulouse. En parallèle à ces deux interventions, les peintures murales de l'église des Cordeliers (Fribourg) - plus de 40000 fragments ! - font l'objet d'un projet d'études pluridisciplinaires, dont Julian James expose les grands axes. L'étude macroscopique des fragments carolingiens de Saint-Remacle de Stavelot est présentée par Emmanuelle Boissard, qui en dégage les caractéristiques techniques tandis que les peintures de Berzé-la-Ville permettent à Juliette Rollier-Hanselman d'établir des comparaisons avec d'autres sites bourguignons.

5 Les études analytiques de pigments bleus et verts correspondent à des problématiques différentes. L'identification des terres vertes dans des fragments romains par Odile Robbe-Cristini pose encore des problèmes expérimentaux, d'autant que l'analyse d'une couche picturale montre, peut-être, la présence d'un liant. Un très petit nombre de sites du haut Moyen Âge est connu pour la France. Bénédicte Palazzo-Bertholon s'est penchée sur les quelques-uns répertoriés présentant des couches picturales bleues, d'origine ou résultant d'une évolution de la couleur. Les pigments identifiés mettent en évidence un changement de nature dont l'interprétation renvoie à l'environnement historique. La villa d'Orbe-Boscéaz (Suisse) a livré à la fois des enduits peints et des pots de pigments. La collaboration d'un archéologue et d'une minéralogiste, Yves Dubois et Jeanne Bonon-Freudiger, a révélé des particularités observées dans les mortiers et les pigments, liées aux techniques d'atelier ainsi que l'utilisation de produits locaux. Enfin les feuilles d'or, dont la présence fut longtemps sousestimée dans les décors romains, peuvent être le siège d'altération dans des conditions spécifiques telles qu'un contexte granitique et marin. Philippe Blanc expose le cas de Mané-Vachen (Bretagne) et établit un lien entre ces altérations et les conditions du battage de l'or pour obtenir la feuille. Michel Fuchs clôt les exposés en montrant à partir de quelques sites suisses les questions restées sans réponse.

6 En marge des interventions "programmées", cet atelier a été enrichi par les remarques et les commentaires de Françoise Perrot, s'interrogeant et nous interrogeant sur le rôle de la lumière sur ces décors peints, lié aux matériaux utilisés et à leur mise en œuvre.

7 Enfin mentionnons la présence de deux enseignants, Alamin Mansouri et Tadeusz Sliwa, du Laboratoire électronique, informatique et image (Le2i) de l'université de Bourgogne implanté à Auxerre, qui ouvre vers une approche d'un type tout à fait différent, mathématique, des couches picturales.

8 La volonté d'une réflexion commune sur une problématique qui touche profondément tous les participants à cet atelier ainsi que la qualité des interventions a permis des échanges nombreux et fructueux. Plusieurs conclusions s'en dégagent.

9 L'importance de la méthodologie de l'étude du décor a été soulignée et conduit à l'élaboration d'un protocole, comme c'est le cas sur certains chantiers. Utilisant un langage de description cohérent et commun, il permettrait des comparaisons et des interprétations pertinentes. Ce protocole devrait recevoir une très large diffusion auprès des différents intervenants sur un chantier. 
10 De même, est apparue la nécessité d'une mise en commun des ressources bibliographiques, en utilisant et exploitant les réseaux déjà existants, enrichis des données de chacun, pour lesquels il faudra définir un langage compréhensif pour tous.

11 Parmi les matériaux constitutifs des décors, le mortier est un élément incontournable, riche d'informations sur la mise en œuvre ou l'utilisation de produits locaux... Son étude devrait être plus généralisée.

12 L'intérêt de cette réunion a été évident pour chacun et a conduit à souhaiter une rencontre en 2010. Un nombre réduit de participants, de l'ordre d'une quinzaine, a paru un bon compromis entre la possibilité de dialogues riches et conviviaux et la présence d'un large éventail d'expériences et de compétences, et devrait déboucher sur une meilleure approche de la diachronie des techniques et des matériaux des décors muraux.

- Paulette HUGON, Méthodologie d'une intervention pluridisciplinaire. Étude technique des peintures murales de la nef de Saint-Savin

- Julian JAMES, Projet d'étude des fragments de peintures murales de l'église des Cordeliers à Fribourg

- Juliette ROLLIER, Étude technique des peintures de Berzé-la-Ville et comparaisons avec d'autres sites de Bourgogne

- Emmanuelle BOISSARD, Les enduits peints de l'abbaye Saint-Remacle de Stavelot (Belgique) : un ensemble de fragments carolingiens

- Odile ROBBE-CRISTINI, Caractérisation de terres vertes utilisées en tant que pigments dans des échantillons archéologiques par spectroscopie Raman : avantages et limitations

- Bénédicte PALAZZO-BERTHOLON, La couleur bleue dans les peintures murales du haut Moyen Âge en France : choix des pigments, ruptures d'approvisionnement et économie de remplacement

- Philippe BLANC, L'altérabilité de la feuille d'or

- Michel fuchs, Analyses des peintures d'Avenches, de Vallon et de quelques sites suisses : les questions restées en suspens

\section{Conclusions : Claude couPRY}

17 Étaient également présents : Annie BLANC, Stéphane BÜTTNER, Marie-Gabrielle CAFFIN, Hélène ERISTON, Alamin MANSOURI, Jean-Charles MEAUDRE, Françoise PERROT, Christian SAPIN et Tadeusz SLIWA. 
INDEX

Mots-clés : peinture murale 